GEOMETRY IN NONLINEAR CONTROL

AND DIFFERENTIAL INCLUSIONS

BANACH CENTER PUBLICATIONS, VOLUME 32

INSTITUTE OF MATHEMATICS

POLISH ACADEMY OF SCIENCES

WARSZAWA 1995

\title{
MINIMA IN CONTROL PROBLEMS WITH CONSTRAINTS
}

\author{
GIANNA STEFANI \\ Dipartimento di Matematica e Applicazioni \\ Via Mezzocannone 8, 80134 Napoli, Italy \\ PIERLUIGI ZEZZA \\ Dipartimento di Matematica Defas \\ Via C. Lombroso 6/17, 50134 Firenze, Italy
}

\begin{abstract}
This paper is devoted to describing second order conditions in the framework of extremal problems, that is, conditions obtained by reducing the optimal control problem to an abstract one in a suitable Banach (or Hilbert) space. The studied problem includes equality constraints both on the end-points and on the state-control trajectory. The second goal is to give a complete description of necessary and sufficient second order conditions for weak local optimality by describing first the associated linear-quadratic problem and then by giving a conjugate point theory for this linear quadratic problem with constraints.
\end{abstract}

1. Introduction. In this paper we describe some recent results on second order necessary and sufficient conditions for weak local minima for an optimal control problem which is characterized by the presence of two types of equality constraints. The first one concerns the end-points of the trajectory, and it is a finite dimensional constraint, while the second one is a time dependent state-control constraint and it can be regarded as being infinite dimensional. We consider the following optimal control problem on the compact interval $J=\left[t_{0}, t_{1}\right]$ :

$$
\text { Minimize } a_{0}\left(\xi\left(t_{0}\right), \xi\left(t_{1}\right)\right)+\int_{t_{0}}^{t_{1}} \ell(t, \xi(t), u(t)) d t
$$

1991 Mathematics Subject Classification: 49K15, 49K27, 93C10, 93C50.

Key words and phrases: optimal control, second order conditions, necessary, sufficient conditions, state-control constraint, conjugate points..

Work partly supported by MURST research grants "Teoria dei sistemi e del controllo" and "Equazioni differenziali ordinarie e applicazioni".

The paper is in final form and no version of it will be published elsewhere. 
over all $\xi$ satisfying the following control problem with constraints:

$$
\begin{array}{cl}
\dot{\xi}(t)=F(t, \xi(t), u(t)), & \text { a.e. } t \in J, \\
a_{i}\left(\xi\left(t_{0}\right), \xi\left(t_{1}\right)\right)=0, & i=1, \ldots, p, \\
\alpha(t, \xi(t), u(t))=0, & \text { a.e. } t \in J,
\end{array}
$$

where the data

$$
\begin{gathered}
F: \mathbf{R} \times \mathbf{R}^{n} \times \mathbf{R}^{m} \rightarrow \mathbf{R}^{n}, \quad \ell: \mathbf{R} \times \mathbf{R}^{n} \times \mathbf{R}^{m} \rightarrow \mathbf{R}, \\
\alpha: \mathbf{R} \times \mathbf{R}^{n} \times \mathbf{R}^{m} \rightarrow \mathbf{R}^{r}, \quad a_{i}: \mathbf{R}^{n} \times \mathbf{R}^{n} \rightarrow \mathbf{R}, \quad i=0, \ldots, p,
\end{gathered}
$$

satisfy regularity assumptions to be specified afterwards.

As far as global minima are concerned, the problem is clear. However sometimes the problem does not guarantee that such a global minimum exists, therefore we are lead to consider local minima. In this case we have to specify which is the topology we are considering.

In the classical calculus of variations, when the control equation is given by $\dot{\xi}=u$, the following cases have been considered.

(i) Strong local minimizers (SLM) the trajectories $\xi$ are considered in $C\left(J, \mathbf{R}^{n}\right)$ with the topology induced by $\|\xi\|_{C}=\max \{\|\xi(t)\|: t \in J\}$.

(ii) Weak local minimizers (WLM) the trajectories $\xi$ are considered in $W^{1, \infty}\left(J, \mathbf{R}^{n}\right)$ with the topology induced by $\|\xi\|_{1, \infty}=\|\xi\|_{C}+\|\dot{\xi}\|_{\infty}=\|\xi\|_{C}+$ $\operatorname{ess} \sup \{\dot{\xi}(t): t \in J\}$.

(iii) $p$-Weak local minimizers $(p$-WLM) the trajectories $\xi$ are considered in $W^{1, p}\left(J, \mathbf{R}^{n}\right)$ with the topology induced by $\|\xi\|_{1, p}=\|\xi\|_{C}+\|\dot{\xi}\|_{p}=\|\xi\|_{C}+$ $\left(\int_{t_{0}}^{t_{1}}(\dot{\xi}(t))^{p} d t\right)^{1 / p}$.

In this case we have $(p \geq 1)$

$$
\mathrm{SLM} \Rightarrow 1-\mathrm{WLM} \Rightarrow \cdots \Rightarrow p-\mathrm{WLM} \Rightarrow \cdots \Rightarrow \text { WLM. }
$$

If we look at the case of the calculus of variations as a control problem, the norm $\|\xi\|_{1, p}$ is equivalent to a norm on the couple (initial point, control) given by $\left\|\xi\left(t_{0}\right)\right\|+\|u\|_{p}$. In the case of a more complicate control equation the two norms are not equivalent. On the other hand the control is a crucial ingredient, therefore the last norm seems more appropriate to define local properties. Notice that usually, and this is our case, the initial point $\xi\left(t_{0}\right)$ and the control map $u$ in a suitable space guarantee the existence and the uniqueness of the solution $\xi$.

For general control systems it is possible to consider the following types of local minimizers.

(i) Strong local minimizers (SLM): the variable is the curve $\xi$ in the space $C\left(J, \mathbf{R}^{n}\right)$.

(ii) Weak local minimizers (WLM): the variable is $\left(\xi\left(t_{0}\right), u\right) \equiv$ (initial point, control) in the Banach space

$$
\mathbf{R}^{n} \times L^{\infty}\left(J, \mathbf{R}^{m}\right)
$$


with the topology $\tau_{\infty}$ induced by $\|(x, u)\|_{\infty} \equiv\|x\|+\|u\|_{\infty}$.

(iii) $p$-Weak local minimizers $\left(p\right.$-WLM): the variable is $\left(\xi\left(t_{0}\right), u\right)$ in the Banach space

$$
\mathbf{R}^{n} \times L^{p}\left(J, \mathbf{R}^{m}\right),
$$

with the topology $\tau_{p}$ induced by $\|(x, u)\|_{p} \equiv\|x\|+\|u\|_{p}$.

Notice that some authors consider WLM as local minimizers in the space $W^{1,1}\left(J, \mathbf{R}^{n}\right) \times L^{\infty}\left(J, \mathbf{R}^{m}\right)$ of the couples $(\xi, u)$ satisfying $(\Xi)$ with the topology induced by $\|(\xi, u)\|=\|\xi\|_{C}+\|\dot{\xi}\|_{1}+\|u\|_{\infty}$, but the minimizers are the same as those we consider.

Remark 1.1. For a general $F$, the control $u \in L^{p}$ may not guarantee the existence of the solution of the differential equation. For example the solutions of $\dot{\xi}(t)=u^{p+1}(t)$ are not defined for every $u \in L^{p}$.

For a general control equation we still have that a SLM is also a WLM, but in general it is not a $p$-WLM, even if we look for local minimizers in $L^{\infty}\left(J, \mathbf{R}^{m}\right)$ with the $\tau_{p}$-topology, as the following example shows.

ExAmple 1.1. Let $a_{0}: \mathbf{R} \rightarrow \mathbf{R}$ be a smooth map which has a local minimum at $x=0$ with $a_{0}(0)=0$ and a minimum at $x=1$ with $a_{0}(1)=-1$. Consider the optimization problem

$$
\text { Minimize } a_{0}(\xi(1))
$$

over all $\xi$ satisfying the following control problem

$$
\dot{\xi}(t)=u^{p+1}(t), \quad \xi(0)=0 .
$$

$\hat{\xi} \equiv 0$ is a SLM. Let us see that $\hat{u} \equiv 0$ is not a local minimizer w.r.t. the topology induced by the $L^{p}$-norm. In fact consider the sequence $\left\{u_{n}\right\}$ defined by

$$
u_{n}(t)= \begin{cases}0 & \text { if } t \leq n^{-n}, \\ \left(\frac{p+1}{n}\right)^{1 /(p+1)} \frac{t^{1 / n}}{t^{1 /(p+1)}} & \text { if } t \geq n^{-n} .\end{cases}
$$

The sequence belongs to $L^{\infty}$ and easy calculations give $\left\|u_{n}\right\|_{p} \rightarrow 0$. On the other hand

$$
\xi_{n} \equiv \xi\left(1, u_{n}\right)=\int_{n^{-n}}^{1} \frac{p+1}{n} t^{-1+\frac{p+1}{n}} d t=1-\frac{1}{n^{p+1}} .
$$

Since $\left\|u_{n}\right\|_{p} \rightarrow 0$ and $\left\|a_{0}\left(\xi_{n}\right)\right\| \rightarrow-1, \hat{u} \equiv 0$ is not a local minimizer w.r.t. the $L^{p}$-norm.

These differences between the calculus of variations and the optimal control depend on $F$ and on its properties. If $F$, for example, is polynomial of degree $p$ in $u$, then, under mild regularity assumptions on $F$, the flow of the system, i.e. the map

$$
\Xi: \mathbf{R}^{n} \times L^{p}\left(J, \mathbf{R}^{m}\right) \rightarrow C\left(J, \mathbf{R}^{n}\right),
$$


which associates to the couple $(x, u)$ the solution of system $(\Xi)$ such that $\xi\left(t_{0}\right)=$ $x$, denoted by $\xi(\cdot, x, u)$, is continuous. Therefore we have

SLM and $F$ polynomial in $u$ of degree $p \Rightarrow p$-WLM.

The relations among all these minimizers in a general control problem need to be investigated.

Our study of second order necessary and sufficient conditions for WLM is in the framework of extremal problems and it is pursued by the following scheme.

The first step amounts to show that after the reduction to an abstract optimization problem and under suitable assumptions, second order conditions can be expressed in Hamiltonian form by the properties of a quadratic form on the set of critical directions of the problem, which is the so called accessory minimization problem, [21]. The second step consists in showing that these properties, namely the nonnegativity or the coerciveness of the form, can be tested by a suitable Jacobi theory, [20].

The study of second order conditions in the calculus of variations goes back to the classical work of A. M. Legendre, C. G. Jacobi and K. Weierstrass who first introduced the accessory minimization problem for the simplest problem in the calculus of variations. Between the wars their results have been extended to more general boundary value problems and to the optimal control setting by G. A. Bliss, M. Hestenes et al. (see e.g. [10]). The presence of state control constraints makes the problem more difficult and most of the literature addressing these constraints considers the case of equality and inequality constraints, so that it is difficult to compare their results with ours. For example some authors impose a constraint qualification assumption that cannot be satisfied by pure equality constraints (see e.g. [13]). A different approach is due to the Russian school which obtains powerful abstract results (for a survey see [11]) whose application to control problems with mixed equality and inequality constraints is stated in [17] but a precise comparison with our results is impossible because the proofs have not been published in an available paper. To obtain stability results for the numerical solution of optimal control problems, in [5] sufficient conditions for weak local optimality are stated for a time-independent problem with inequality and equality constraints only on the control. Moreover they assume that the linearized system is controllable and that the reference trajectory satisfies the maximum principle. The study of second order conditions through the accessory minimization problem is a subject of active research and the quoted papers are just a sample of different approaches and do not represent an exhaustive bibliography.

The original Jacobi results on conjugate points are about the simplest problem in the calculus of variations, where both end-points are fixed. Until the crucial work by M. Hestenes and M. Morse, [9], [16], the improvements have concerned only the regularity of the data and of the optimal solution, while in their results an index theory for quadratic forms in Hilbert spaces is developed and the connection between the index and the conjugate (or focal) points is explained. In 
optimal control problems the situation is more complicated than in the calculus of variations because the quadratic form can be semidefinite (and non-positive) on an interval, called focal interval in [15] or table in [4]. The quoted results and our analysis concern the case when the strengthened Legendre-Clebsch condition holds, while the singular case has been analyzed with details in [1]. In all these papers the case when both end-points are variable is never considered, except the special case of periodic boundary conditions, [14]. This problem has been first analyzed in [25], [26] where, under suitable controllability assumptions, the authors introduce the concept of coupled point and state the corresponding necessary conditions.

In $[27,20]$ an abstract Jacobi theory obtained by merging some ideas of Hestenes and Poincaré is presented and it is applied to LQ-optimal control problem with constraints. The conjugate points can be characterized by the solutions of the Jacobi system which satisfy suitable transversality conditions. The abstract theory points out that the different definitions of conjugate, focal and coupled points can be seen as corresponding to the same object in different situations. For this reason we go back to the original name of conjugate point, including in this definition all the previous mentioned cases.

As far as strong local minima are concerned we want to mention, besides the classical results of K. Weierstrass, the results in [24] where sufficient conditions for weak and strong local minima are given for a problem in the calculus of variations with separate constraints on the end-points but without other restrictions on the control. The approach refers to the properties of the solutions of the associated Riccati equation. In [2] sufficient conditions are given for an optimal control problem with control taking values in a given set $U$ and with fixed end-points, the approach uses tools from symplectic geometry and extends the notion of field of extremals.

The main results are in the next section, while a sketch of the proofs is in Section 3. A complete description of the problem and complete proofs are in [21, $22]$, previous versions of the results can be found in $[18,19,20,27]$.

2. Main results. Let us first introduce some notations and definitions needed to describe properly the assumptions and the main results.

$X, Y, Z$ are Banach spaces with norm $\|\cdot\|$. Let $\phi: X \rightarrow Y$ be a $C^{2}$ map, we write $D^{i} \phi(x)$ for the $i$ th-Fréchet derivative of the map $\phi$ evaluated at the point $x \in X$, by definition $D^{0} \phi \equiv \phi$ and $D \phi(x) \in \mathcal{L}(X, Y), D^{2} \phi(x) \in \mathcal{L}^{2}(X, Y)$. For $\Gamma \in \mathcal{L}^{2}(X, Y)$ we will write

$$
\Gamma(x)^{2} \equiv \Gamma(x, x) .
$$

If $X \equiv X_{1} \times \cdots \times X_{s}$, with $X_{1}, \ldots, X_{s}$ normed spaces, we denote by $D_{i} \phi(x)$ the Fréchet derivative of $\phi$ with respect to the $i^{\text {th }}$-variable and by $D_{i j}^{2} \phi(x) \equiv$ $D_{i} \circ D_{j} \phi(x) \in \mathcal{L}^{2}\left(X_{i} \times X_{j}, Y\right)$. 
The next definition will be used to describe the main regularity assumption (Assumption 2.1) which is a strengthening of the usual Carathéodory-type assumption. This hypothesis is related to the regularity of the dependence of the solution of the system on the control, see $[8,21]$.

Definition 2.1 (see [8]). Assume that $X, Y$ are finite dimensional vector spaces. We will say that the map $G: \mathbf{R} \times X \rightarrow Y$ is quasi- $C^{k}$ if it satisfies the following:

(i) for each $t \in \mathbf{R}$ the map $x \mapsto G(t, x)$ is $C^{k}$,

(ii) the maps $D_{2}^{i} G$ are locally essentially bounded and measurable in their variables, for $i=0, \ldots, k$.

Moreover we will say that the map $G$ is uniformly quasi- $C^{k}$ if

(iii) the map $D_{2}^{k} G$ is continuous in $x$ uniformly with respect to $t$ in any compact interval $J$, i.e. for all $x_{0} \in X, \epsilon>0$, there exists $\delta>0$ such that

$$
\left\|x-x_{0}\right\| \leq \delta \Longrightarrow\left\|D_{2}^{k} G(t, x)-D_{2}^{k} G\left(t, x_{0}\right)\right\| \leq \epsilon, \quad \text { a.e. } t \in J .
$$

It is straightforward to prove that if a function is quasi- $C^{k}$ then it is uniformly quasi- $C^{k-1}$.

Since we are interested in local properties, we could assume that the domains of all the maps are not the whole space but just open sets; we prefer the above notation to emphasize the space where we are working.

The data are assumed to satisfy the following regularity assumptions:

Assumption 2.1. The maps $F: \mathbf{R} \times \mathbf{R}^{n} \times \mathbf{R}^{m} \rightarrow \mathbf{R}^{n}$ and $\ell: \mathbf{R} \times \mathbf{R}^{n} \times \mathbf{R}^{m} \rightarrow \mathbf{R}$ are quasi- $C^{2}$, the map $\alpha: \mathbf{R} \times \mathbf{R}^{n} \times \mathbf{R}^{m} \rightarrow \mathbf{R}^{r}$ is uniformly quasi-C $C^{2}$ and the maps $a_{i}: \mathbf{R}^{n} \times \mathbf{R}^{n} \rightarrow \mathbf{R}, \quad i=0, \ldots, p$, are $C^{2}$.

In the following we will consider a given $\left(x_{0}, \hat{u}\right)$ which satisfies the constraints and we denote by $\hat{\xi}$ the corresponding solution of $(\Xi)$ and by $x_{1}$ the value $\hat{\xi}\left(t_{1}\right)$. As usual we denote by " " the evaluation along the reference objects and by "T" the transpose.

An assumption which will play a crucial role, concerns the infinite dimensional constraint $\alpha$.

Assumption 2.2. The constraint $\alpha$ satisfies the following rank condition at $\left(x_{0}, \hat{u}\right)$ :

$$
\operatorname{det}\left(D_{3} \hat{\alpha}(t) D_{3} \hat{\alpha}(t)^{\top}\right) \geq k>0
$$

for some positive $k \in \mathbf{R}$.

This assumption allows us to give an explicit (through the data) expression of the modified Hamiltonian associated to the constrained problem. Let

$$
\begin{gathered}
A(t) \equiv D_{2} \hat{F}(t), \quad B(t) \equiv D_{3} \hat{F}(t), \quad B_{0}(t) \equiv D_{3} \hat{\ell}(t), \\
C(t) \equiv D_{2} \hat{\alpha}(t), \quad D(t) \equiv D_{3} \hat{\alpha}(t) .
\end{gathered}
$$


For sake of simplicity we will denote by $\nabla$ the derivative with respect to the coupled variables $(x, w) \in \mathbf{R}^{n} \times \mathbf{R}^{m}$, so that for example $\nabla \hat{\alpha}(t)=(C(t), D(t))$.

The second order conditions will hold on the space of critical directions, i.e. the space of couples $(x, u)$ which satisfy the following system obtained by linearizing $(\Xi)$ and the constraints along the reference trajectory:

$$
\begin{gathered}
\dot{\xi}_{L}(t)=A(t) \xi_{L}(t)+B(t) u(t), \quad \xi_{L}\left(t_{0}\right)=x, \\
C(t) \xi_{L}(t)+D(t) u(t)=0, \\
D a_{i}\left(x_{0}, x_{1}\right)\left(x, \xi_{L}\left(t_{1}\right)\right)=0, \quad i=1, \ldots, p .
\end{gathered}
$$

We denote the solutions of equation (1) by $\xi_{L}(\cdot, x, u)$. Assumption 2.2 assures the existence of a right inverse of $D(t)$ which can be taken as

$$
D^{\sharp}(t) \equiv D^{\top}(t)\left(D(t) D^{\top}(t)\right)^{-1} .
$$

The next two theorems give first and second order necessary or sufficient weak local optimality conditions for this kind of constraints, by means of the Hamiltonian $\mathcal{H}: J \times\left(\mathbf{R}^{n}\right)^{*} \times \mathbf{R} \times \mathbf{R}^{n} \times \mathbf{R}^{m} \rightarrow \mathbf{R}$ modified to take into account the infinite dimensional constraint and defined by

$$
\begin{aligned}
& \mathcal{H}\left(t, \omega, \omega_{0}, x, w\right) \\
& \quad=\omega\left(F(t, x, w)-B(t) D^{\sharp}(t) \alpha(t, x, w)\right)+\omega_{0}\left(\ell(t, x, w)-B_{0}(t) D^{\sharp}(t) \alpha(t, x, w)\right) .
\end{aligned}
$$

The first result concerns necessary optimality conditions.

TheOREM 2.1. Let Assumptions 2.1, 2.2 hold and assume that $\left(x_{0}, \hat{u}\right)$ is a weak local minimizer for the optimal control problem, then there exist $\left(\lambda_{0}, \ldots, \lambda_{p}\right) \neq 0$ with $\lambda_{0} \geq 0$ and a solution $\hat{p}$ of the adjoint equation (4) and of the transversality conditions (5)

$$
\begin{aligned}
& -\dot{p}(t)=D_{4} \mathcal{H}\left(t, p(t), \lambda_{0}, \hat{\xi}(t), \hat{u}(t)\right), \\
& \left(-p\left(t_{0}\right), p\left(t_{1}\right)\right)=\sum_{i=0}^{p} \lambda_{i} D a_{i}\left(x_{0}, x_{1}\right),
\end{aligned}
$$

such that

$$
D_{5} \hat{\mathcal{H}}(t)=\left(\hat{p}(t) B(t)+\lambda_{0} B_{0}(t)\right)\left(I d-D^{\sharp}(t) D(t)\right)=0 .
$$

Assume moreover that the above multiplier $\left(\lambda_{0}, \ldots, \lambda_{p}\right)$ is unique up to a positive constant, then for each $(x, u)$ satisfying the linearized system $(1),(2),(3)$ we have

$$
\begin{aligned}
& \sum_{i=0}^{p} \lambda_{i} D^{2} a_{i}\left(x_{0}, x_{1}\right)\left(\left(x, \xi_{L}\left(t_{1}, x, u\right)\right)\right)^{2} \\
& \qquad+\int_{t_{0}}^{t_{1}} \nabla^{2} \hat{\mathcal{H}}(s)\left(\left(\xi_{L}(s, x, u), u(s)\right)\right)^{2} d s \geq 0 .
\end{aligned}
$$

The above theorem is given under the assumption that the multiplier associated with the finite dimensional part (end-points cost and constraints) is unique 
up to a positive constant. In the literature there has also been considered the case when this assumption is dropped [7,23]. Their results specialized to our case give trivial conditions in the following sense. They would say that for each critical direction $(x, u)$ there is a multiplier $\left(\lambda_{0}, \ldots, \lambda_{p}\right)$ such that $(6)$ and $(7)$ hold. It is a consequence of Corollary 4.1 in [21] that this is true independently of the cost and it depends only on the existence of independent multipliers.

The second result concerns second order sufficient conditions. It does not require the uniqueness of the multiplier and it is stated under stronger regularity assumptions on $F$ and $\ell$.

TheOREM 2.2. Let Assumptions 2.1, 2.2 hold. Assume moreover that $F$ is uniformly quasi- $C^{2}$ and

(i) there exist $\left(\lambda_{0}, \ldots, \lambda_{p}\right) \neq 0 \in\left(\mathbf{R}^{p+1}\right)^{*}$ with $\lambda_{0} \geq 0$ and a solution $\hat{p}$ of the adjoint equation (4) and of the transversality conditions (5) for which the first order conditions (6) are satisfied,

(ii) there is $K>0$ such that for each $(x, u)$ satisfying the linearized system (1), (2), (3)

$$
\begin{aligned}
& \sum_{i=0}^{p} \lambda_{i} D^{2} a_{i}\left(x_{0}, x_{1}\right)\left(\left(x, \xi_{L}\left(t_{1}, x, u\right)\right)\right)^{2} \\
& \quad+\int_{t_{0}}^{t_{1}} \nabla^{2} \hat{\mathcal{H}}(s)\left(\left(\xi_{L}(s, x, u), u(s)\right)\right)^{2} d s \geq K\|(x, u)\|_{2}^{2}
\end{aligned}
$$

then $\left(x_{0}, \hat{u}\right)$ is a weak local minimizer for the optimal control problem.

Remark 2.1. Although Theorems 2.1 and 2.2 are stated without any normality assumption, the abnormal case, i.e. $\lambda_{0}=0$, has a particular meaning. Let us first remark that the multiplier is normal and unique if and only if the point $\left(x_{0}, \hat{u}\right)$ is regular for the constraints and if and only if the following input-output system is controllable at time $t_{1}$ (see Lemma 5.3 in [21])

$$
\begin{gathered}
\dot{\eta}(t)=\left(A(t)-B(t) D^{\sharp}(t) C(t)\right) \eta(t)+B(t)\left(I d-D^{\sharp}(t) D(t)\right) u(t), \quad \eta\left(t_{0}\right)=x, \\
y_{i}(t)=D a_{i}\left(x_{0}, x_{1}\right)(x, \eta(t, x, u)), \quad i=1, \ldots, p,
\end{gathered}
$$

with this we mean that the input-output map $(x, u) \mapsto\left(y_{1}\left(t_{1}\right), \ldots, y_{p}\left(t_{1}\right)\right)$ is surjective.

Assume that the multiplier $\left(\lambda_{0}, \ldots, \lambda_{p}\right)$ is unique. If the point $\left(x_{0}, \hat{u}\right)$ is not regular for the constraints, then the multiplier is abnormal and the statements do not involve the cost $a_{0}$. Nevertheless, if (7) is not satisfied, then the reference point may be an extremum only for a cost $a_{0}$ for which there are independent multipliers satisfying the first order conditions.

The uniqueness of the multiplier does not play any role in the sufficient conditions but if Theorem 2.2 holds for an abnormal multiplier then the reference point $\left(x_{0}, \hat{u}\right)$ is an isolated admissible point. 
Both the necessary and the sufficient conditions in Theorems 2.1, 2.2 are stated through a linear quadratic problem which can be described in the following way. Let

$$
\begin{gathered}
P(t) \equiv D_{44} \hat{\mathcal{H}}(t), \quad Q(t) \equiv D_{45} \hat{\mathcal{H}}(t), \quad R(t) \equiv D_{55} \hat{\mathcal{H}}(t), \\
\Gamma=\sum_{i=0}^{p} \lambda_{i} D^{2} a_{i}\left(x_{0}, x_{1}\right) .
\end{gathered}
$$

The above considered quadratic form can be written as

$$
\begin{aligned}
J(x, u)^{2}= & \frac{1}{2} \Gamma\left(x, \xi_{L}\left(t_{1}\right)\right)^{2} \\
& +\frac{1}{2} \int_{t_{0}}^{t_{1}}\left(P(s)\left(\xi_{L}(s)\right)^{2}+2 Q(s)\left(\xi_{L}(s), u(s)\right)+R(s)(u(s))^{2}\right) d s,
\end{aligned}
$$

where $(x, u)$ is a critical direction, i.e. satisfies (1), (2) and a boundary condition as

$$
N\left(x, \xi_{L}\left(t_{1}\right)\right)=0,
$$

with $N \in \mathcal{L}\left(\mathbf{R}^{2 n}, \mathbf{R}^{p}\right)$. Under our regularity assumptions the quadratic form $J$ is defined and continuous also on the Hilbert space

$$
\mathbf{R}^{n} \times L^{2}\left(\left[t_{0}, t_{1}\right], \mathbf{R}^{m}\right)
$$

endowed with the standard product norm. Our second goal is to give necessary and sufficient conditions for the quadratic form $J$ to be coercive or nonnegative on the subspace of the couples $(x, u)$ satisfying equation (1) with the constraints (2) and (8).

Quadratic forms in Hilbert spaces can be identified with linear operators and we will use the same notation, so that for example $P(t)$ is a quadratic form, a linear operator and a matrix. Thanks to Assumption 2.2 we can define

$$
\Pi_{1}=I d-D^{\sharp} D, \quad \Pi_{2}=D^{\sharp} D .
$$

The other main assumption we are going to make is the so called "strengthened Legendre-Clebsch condition" (see [15, 20]), which can be stated as

Assumption 2.3. There is $k>0$ such that

$$
\Pi_{1} R \Pi_{1}+\Pi_{2} \geq k I d
$$

It is not difficult to see that if the feed-back control

$$
u=-D^{\sharp} C \xi_{L}
$$

is used, then the constraint (2) is satisfied independently of the starting point of the trajectory. We choose this feed-back control as the reference one and we define the conjugate points by means of a family of problems which are parametrized by the time $c \in\left[t_{0}, t_{1}\right]$. These problems are obtained by taking the admissible couples in the subspace $H_{c}$ corresponding to the controls which are equal to the 
reference one on $\left[c, t_{1}\right]$. On this time interval the corresponding solution of the control system (1) can be expressed through the solution $\Phi(t, c)$ of the matrix equation

where

$$
\dot{\Phi}(t)=\bar{A}(t) \Phi(t), \quad \Phi(c)=I d,
$$

$$
\bar{A} \equiv A-B D^{\sharp} C .
$$

The problems of this family can be considered as problems on $\left[t_{0}, c\right]$ with cost $J_{c}=J_{\mid H_{c}}$ and where the end-points cost and constraints are given by

$$
\begin{gathered}
N_{c}(x, y)=N\left(x, \Phi\left(t_{1}, c\right) y\right), \\
\Gamma_{c}(x, y)^{2}=\Gamma\left(x, \Phi\left(t_{1}, c\right) y\right)^{2}+\left(\int_{c}^{t_{1}} \Phi^{\top}(s, c) \bar{P}(s) \Phi(s, c) d s\right)(y)^{2},
\end{gathered}
$$

where

$$
\bar{P} \equiv P-2 C^{\top}\left(D^{\sharp}\right)^{\top} Q+C^{\top}\left(D^{\sharp}\right)^{\top} R D^{\sharp} C .
$$

In particular, when $c=t_{0}$, we consider the restriction of the quadratic form $J$ to the subspace corresponding to the control which is feed-back on the whole $\left[t_{0}, t_{1}\right]$, this subspace can be identified with the subspace of $\mathbf{R}^{n}$ given by

$$
H_{t_{0}} \equiv\left\{x \in \mathbf{R}^{n}: N_{t_{0}}(x, x)=0\right\} .
$$

Notice that $H_{t_{0}}$ may be nontrivial if no end-point is fixed. The restriction of $J$ to $H_{t_{0}}$ can be written as the finite dimensional quadratic form

$$
J_{t_{0}}: x \mapsto \frac{1}{2} \Gamma_{t_{0}}(x, x)^{2} .
$$

Since our goal is to state necessary and sufficient conditions in terms of "conjugate points" we will need the Jacobi system associated to this problem. The presence of the functional constraint in (2) modifies the usual system.

From Assumption 2.3 we can deduce that $\Pi_{1} R \Pi_{1}+\Pi_{2}$ has an inverse. Set

$$
S=\left(\Pi_{1} R \Pi_{1}+\Pi_{2}\right)^{-1} \Pi_{1}, \quad \bar{Q} \equiv Q-R D^{\sharp} C
$$

and define the Hamiltonian $\mathcal{K}: \mathbf{R} \times\left(\mathbf{R}^{n}\right)^{*} \times \mathbf{R}^{n} \rightarrow \mathbf{R}$ by

$$
\begin{aligned}
\mathcal{K}(t, \omega, x)= & \frac{1}{2}\left(-\left(\bar{Q}^{\top}(t) S(t) \bar{Q}(t)-\bar{P}(t)\right)(x)^{2}-S(t)\left(B^{\top}(t) \omega^{\top}\right)^{2}\right) \\
& +\omega(\bar{A}(t)-B(t) S(t) \bar{Q}(t)) x .
\end{aligned}
$$

Notice that the presence of extra-terms in the Hamiltonian depends on the pointwise constraints on the state, in fact they disappear if $C=0$.

By means of the solutions of the Hamiltonian system associated to $\mathcal{K}$ we can characterize the extremals for our problem.

Definition 2.2. An absolutely continuous function

$$
(\zeta, \mu):\left[t_{0}, t_{1}\right] \rightarrow \mathbf{R}^{n} \times\left(\mathbf{R}^{n}\right)^{*}
$$


is called an extremal if it is a solution of the Jacobi system

$$
\dot{\zeta}(t)=D_{2} \mathcal{K}(t, \mu(t), \zeta(t)) \quad \dot{\mu}(t)=-D_{3} \mathcal{K}(t, \mu(t), \zeta(t)) .
$$

An extremal is said to be $c$-transversal if it is admissible, i.e.,

$$
N_{c}\left(\zeta\left(t_{0}\right), \zeta(c)\right)=0,
$$

and it satisfies the following transversality conditions:

$$
\left(-\mu\left(t_{0}\right), \mu(c)\right)=\left\langle\Gamma_{c}\left(\zeta\left(t_{0}\right), \zeta(c)\right), \cdot\right\rangle+\sigma N_{c}, \quad \text { for some } \sigma \in\left(\mathbf{R}^{k}\right)^{*} .
$$

A $c$-transversal extremal will be called nontrivial if its state component $\zeta$ is not identically zero on $\left[t_{0}, c\right]$.

Notice that the above definition holds also for $c=t_{0}$, and in this case it means simply that there exists a critical point $x$ of $J_{t_{0}}$ restricted to $H_{t_{0}}$.

In order to distinguish between the points $c$ where $J_{c}$ becomes semi-definite and those where $J_{c}$ becomes indefinite we need to consider $c$-transversal extremals with $\zeta$-subarcs corresponding to the reference control.

Definition 2.3. A $c$-transversal extremal $(\zeta, \mu)$ is said to be degenerate on the interval $[\alpha, \beta]$ containing $c$ if

$$
\Pi_{1}(t)\left(\bar{Q}(t) \zeta(t)+B^{\top}(t) \mu^{\top}(t)\right)=0, \quad t \in[\alpha, \beta]
$$

or, equivalently, for $t \in[\alpha, \beta]$

$$
\dot{\zeta}(t)=\bar{A}(t) \zeta(t), \quad \dot{\mu}(t)=-\mu(t) \bar{A}(t)-\zeta^{\top}(t) \bar{P}(t) .
$$

We can now introduce the definition of conjugate and semi-conjugate point. The two definitions coincide in the calculus of variations when the right end-point is fixed because in this case all the degenerate $c$-transversal extremals are trivial.

Definition 2.4. A point $c \in\left[t_{0}, t_{1}\right]$ is called semi-conjugate to zero if there exists a nontrivial $c$-transversal extremal $(\zeta, \mu)$.

A point $c \in\left[t_{0}, t_{1}\right)$ is called conjugate to zero if there exists a non trivial $c$-transversal extremal $(\zeta, \mu)$ which cannot be continued as a degenerate extremal on $\left[c, c_{1}\right], c_{1}>c$.

We are now able to state the results corresponding to the classical Jacobi necessary and sufficient conditions. Under Assumptions 2.2 and 2.3 the following hold

THEOREM 2.3. The quadratic form $J$ is nonnegative if and only if $J_{t_{0}}$ is nonnegative on $H_{t_{0}}$ and there is no point $c \in\left[t_{0}, t_{1}\right)$ conjugate to zero.

THEOREM 2.4. The quadratic form $J$ is coercive if and only if $J_{t_{0}}$ is positive on $H_{t_{0}}$ and there is no point $c \in\left(t_{0}, t_{1}\right]$ semi-conjugate to zero.

The above results include all the quoted results concerning conjugate, focal and coupled points. Theorems 2.1, 2.2 with Theorems 2.3, 2.4 give a complete characterization of WLM in terms of accessory minimization problem and of conjugate points. 
3. Abstract results. In this section we will briefly sketch the approach used in proving the theorems stated in Section 2. In order to prove Theorems 2.1 and 2.2 in the spirit of obtaining extremality conditions for an abstract problem we proceed in the following way.

Step 1. Reduce the problem to an abstract optimization problem on $E=$ $\mathbf{R}^{n} \times L^{\infty}$ as follows. Let us first remark that by adding an extra coordinate with equation $\dot{x}^{0}=\ell(t, x, u)$ and with initial condition $x^{0}\left(t_{0}\right)=0$ and by adding to the objective function the extra cost $x^{0}\left(t_{1}\right)$, the problem can be transformed into the Mayer form. Then, without loss of generality, we can assume that $\ell=0$. The reduction can be obtained by defining for $i=0, \ldots, p, \phi_{i}: E \rightarrow \mathbf{R}$ by

$$
\phi_{i}(x, u)=a_{i}\left(x, \xi\left(t_{1}, x, u\right)\right), \quad i=0, \ldots, p,
$$

and $\psi: E \rightarrow F \equiv L^{\infty}\left(J, \mathbf{R}^{r}\right)$ by

$$
\psi(x, u)(t)=\alpha(t, \xi(t, x, u), u(t)) .
$$

The transformed problem is the following

$$
\text { Minimize } \phi_{0}(e) \text { subject to the constraint } \chi(e)=0 \text {, }
$$

where $\chi \equiv\left(\phi_{1}, \ldots, \phi_{p}\right)+\psi$. The study of the problem will be pursued through an analysis of the range of the map

$$
\tilde{\chi} \equiv \phi_{0}+\chi: E \rightarrow Z \equiv \mathbf{R}^{p+1} \oplus F .
$$

The first component of $\tilde{\chi}$ is the cost and it has a special role, for this reason we indicate with $\mathbf{z}_{\mathbf{0}}$ the unit vector of the cost axis, i.e. the vector in $Z$ which has the first component equal to one and all the others equal to zero. A point $e=(x, u) \in E$ satisfies the constraints if and only if it has image on the straight line through the origin, parallel to $\mathbf{z}_{\mathbf{0}}$, these points will be called admissible. The point $e$ is said to be regular for the constraints $\chi$ if and only if $D \chi(e)$ is onto. An element $\Lambda \in Z^{*}$, called multiplier, is said to be normal if and only if $\Lambda \mathbf{z}_{\mathbf{0}} \neq 0$, that is, its cost component is not zero.

An admissible reference couple $\hat{e} \equiv\left(x_{0}, \hat{u}\right)$ is a weak local minimizer for the original problem if and only if there exists a neighborhood $\Theta$ of $\hat{e}$ in $E$ such that

$$
\tilde{\chi}(\Theta) \cap\left\{\tilde{\chi}(\hat{e})-a \mathbf{z}_{\mathbf{0}}: a>0\right\}=\emptyset .
$$

The properties of the range of $\tilde{\chi}$ will be described by the first and second derivatives of $\tilde{\chi}$ at the reference point $\hat{e} \in E$. In particular in the normal case the second order conditions depend only on the so called Hessian of $\tilde{\chi}$ while in the abnormal one they depend only on the Hessian of $\chi$ (see Remark 3.1). Recall that the Hessian is the the restriction of the second derivative to the kernel of the first one modulo the range of the first derivative. Set

$$
\tilde{\chi}^{\prime} \equiv D \tilde{\chi}(\hat{e}), \quad \tilde{\chi}^{\prime \prime} \equiv D^{2} \tilde{\chi}(\hat{e}),
$$

the same notation will be used also for other maps. 
S tep 2. Prove that under Assumptions 2.1, 2.2 we obtain that the map $\tilde{\chi}$ satisfies

(i) $\tilde{\chi}$ is a $C^{2}$ map,

(ii) $\psi^{\prime}$ is onto and it has a continuous right inverse $\psi^{\sharp}: F \rightarrow E$.

The regularity of $\tilde{\chi}$ is obtained by proving that the flow of the system has a $C^{2}$ dependence on the initial state and control in the appropriate function spaces. A crucial role is played by the assumption on the constraints. Assumption 2.2 is equivalent to assuming that the reference point is a regular point for the infinite dimensional constraint $\psi$, i.e. $D \psi(\hat{e})$ is onto, [19]. It allows us to reduce the constraint in the abstract problem to a finite dimensional one and it assures that the multiplier associated with the state-control constraint belongs to $L^{\infty}$ (see Lemma 4.1 in [21]). In fact let us consider a complement $Z_{2}$ of $\operatorname{Im}\left(\phi_{1}^{\prime}, \ldots, \phi_{p}^{\prime}\right)_{\mid K e r \psi^{\prime}}$ in $\mathbf{R}^{p+1}$, if the properties (i) and (ii) hold, then $\operatorname{Im} \tilde{\chi}^{\prime}$ is closed, $E=\operatorname{Im} \tilde{\chi}^{\prime} \oplus Z_{2}$ and there is $\tilde{\chi}^{\sharp}: Z \rightarrow E$ such that

$$
\tilde{\chi}_{\mid Z_{2}}^{\sharp}=0, \quad \tilde{\chi}^{\prime} \tilde{\chi}_{\mid \operatorname{Im}}^{\sharp} \tilde{\chi}^{\prime}=I d .
$$

Moreover $E=\operatorname{Ker} \tilde{\chi}^{\prime} \oplus \operatorname{Im} \tilde{\chi}^{\sharp}$.

Step 3. Prove the following lemma (an abstract version of Theorem 2.1) for maps which satisfy (i) and (ii).

\section{Lemma 3.1. Assume that}

1) there exists a neighborhood $\Theta$ of $\hat{e}$ such that (15) holds,

2) codim $\operatorname{Im} \tilde{\chi}^{\prime}=1$,

then there exists a nonzero multiplier $\Lambda \in Z^{*}$ such that

(i) $\Lambda \tilde{\chi}^{\prime}=0$,

(ii) $\Lambda \mathbf{z}_{0} \geq 0$,

(iii) $\Lambda \tilde{\chi}^{\prime \prime}(e)^{2}$ is nonnegative on $\operatorname{Ker} \chi^{\prime}$.

To derive the sufficient conditions it is crucial to consider different norms on $L^{\infty}$, because a coerciveness condition on the second derivative is needed but it cannot be imposed on $L^{\infty}$ endowed with its norm because it is not isomorphic to a Hilbert space. To state sufficient conditions for (15) to hold, we are now going to consider the Banach space $E$ endowed also with another, possibly different, norm $\|\cdot\|_{2}$ and we denote by $\tau$ the topology under which $E$ is a Banach space and by $\tau_{2}$ the other. Let us underline that it is necessary that the completion of $E$ under $\tau_{2}$ is a Hilbert space otherwise no continuous positive quadratic form on $\left(E, \tau_{2}\right)$ could be coercive.

The next lemma provides an abstract framework to prove sufficient conditions for weak local optimality for our optimization problem. Notice that a different kind of regularity involving both $\tau$ and $\tau_{2}$ topologies is needed. For this reason the regularity Assumption 2.1 need to be strengthened to prove sufficient conditions, and it cannot be weakened as it is shown by Example 3.2 in [21]. 
LEMMA 3.2. Assume that

1) $\tilde{\chi}^{\sharp} D \tilde{\chi}:(E, \tau) \rightarrow \mathcal{L}\left(\left(E, \tau_{2}\right),\left(E, \tau_{2}\right)\right)$ is continuous.

Assume moreover that there exists a multiplier $\Lambda \in Z^{*}$ such that

2) $\Lambda D^{2} \tilde{\chi}:(E, \tau) \rightarrow \mathcal{L}^{2}\left(\left(E, \tau_{2}\right), \mathbf{R}\right)$ is continuous,

3) $\Lambda \tilde{\chi}^{\prime}=0$,

4) $\Lambda \mathbf{z}_{0} \geq 0$

5) $\Lambda \tilde{\chi}^{\prime \prime}$ is coercive on $K$ er $\chi^{\prime}$ with respect to the norm $\|\cdot\|_{2}$.

Then there exists a neighborhood $\Theta$ of $\hat{e}$ in $(E, \tau)$ such that

$$
\tilde{\chi}(\Theta) \cap\left\{\tilde{\chi}(\hat{e})-a \mathbf{z}_{\mathbf{0}}: a>0\right\}=\emptyset .
$$

Remark 3.1. It is important to stress the difference between the normal case $\left(\Lambda \mathbf{z}_{\mathbf{0}} \neq 0\right)$ and the abnormal one $\left(\Lambda \mathbf{z}_{0}=0\right)$. In the normal case $\operatorname{Ker} \tilde{\chi}^{\prime}=$ $\operatorname{Ker} \chi^{\prime}$ and the second order optimality conditions concern the quadratic form $\Lambda \tilde{\chi}_{\mid \text {Ker } \tilde{\chi}^{\prime}}^{\prime \prime}$ which depends on the Hessian of $\tilde{\chi}$. In the abnormal case $\Lambda \in\left(\mathbf{R}^{p} \oplus\right.$ $F)^{*}$ and the quadratic form is $\Lambda \chi^{\prime \prime} \mid$ Ker $\chi^{\prime}$ which depends only on the Hessian of the constraints $\chi$. In this last case the optimality conditions give essentially informations on the constraints. In fact as far as the necessary conditions are concerned the information we have is that if $\operatorname{codim} \operatorname{Im} \chi^{\prime}=1$ and $\Lambda \chi^{\prime \prime}{ }_{\text {Ker }} \chi^{\prime}$ is indefinite, then $\hat{e}$ may be an extremum only for a cost satisfying codim $\operatorname{Im} \tilde{\chi}^{\prime}=2$. If the sufficient conditions hold true for an abnormal multiplier, then they hold true for any cost so that the point $\hat{e}$ is isolated among the admissible points.

Step 4. We prove the equivalence between the existence of a multiplier $\Lambda$ satisfying 3 ) and 4) of Lemma 3.2 and the existence of an adjoint covector satisfying (4), (5) and (6). Finally we prove that $\Lambda \tilde{\chi}^{\prime \prime}$ coincides with the left hand part of $(7)$.

In order to state the necessary and sufficient conditions for the quadratic form $J$ to be nonnegative or coercive in terms of the Jacobi system and of conjugate points we use the results in [27]. The abstract theory analyzes the coerciveness of an elliptic form (for the definition see [15]) on a Hilbert space $H$ by means of a family of subspaces depending on a parameter $c$. The family must satisfy the following "continuity" properties.

Definition 3.1. We will say that a family $H_{c}, c \in\left[c_{0}, c_{1}\right]$, of closed subspaces of $H$ defines a Jacobi condition if it has the following properties:

(i) for $c_{0} \leq c \leq d \leq c_{1}, H_{c} \subseteq H_{d}$, and $H_{c_{1}}=H$,

(ii) for $d \in\left(c_{0}, c_{1}\right], c l\left(\bigcup_{c_{0} \leq c<d} H_{c}\right)=H_{d}$,

(iii) for $d \in\left[c_{0}, c_{1}\right), \bigcap_{d<c \leq c_{1}} H_{c}=H_{d}$.

Let $q$ be a weakly continuous positive quadratic form on $H$, the study of the form $J$ is pursued through the "value function" $V:\left[c_{0}, c_{1}\right] \rightarrow(-\infty,+\infty]$ defined as

$$
V(c)=\min _{e \in \Omega} J_{\mid H_{c}}(e)
$$


where

$$
\Omega=\left\{e \in H: q(e)^{2}=1\right\} \text { and } V(c)=+\infty \quad \text { if } \quad \Omega \cap H_{c}=\emptyset .
$$

The main result in [27] is a kind of "continuation principle" which states that if the value function $V$ at level $c=c_{0}$ is positive and it does not become zero along the family then at the final level $c_{1}$, which corresponds to the whole space, it is still positive and this implies that the form is coercive. This goal can be reached by the following

TheOREM 3.1 (see [27]). Assume that $J$ is elliptic on $H$. Then the function $V$ is nonincreasing and it is continuous as an extended function

$$
V:\left[c_{0}, c_{1}\right] \rightarrow \mathbf{R} \cup\{+\infty\} .
$$

It is now a matter of elementary arguments to establish the "continuation principle" we need. The properties of $V$ yield that $J$ is coercive on $H$ if and only if $J$ is positive on $H_{c_{0}}$ and there is no point $c \in\left(c_{0}, c_{1}\right]$ at which $V$ becomes zero. Moreover $J$ is nonnegative on $H$ if and only if $J$ is nonnegative on $H_{c_{0}}$ and there is no point $c \in\left[c_{0}, c_{1}\right)$ at which $V$ changes sign.

In our specific case these abstract results can be applied in the following way.

Step 1. We perform the feed-back change of control

$$
u \mapsto u+D^{\sharp} C \xi_{L},
$$

which changes the reference feed-back control (9) into the zero one. In this way we change our original LQ-control problem into a simpler one. Namely, thanks to Assumption 2.2, our quadratic form is equivalent to another one with a functional constraint which does not depend on the state (i.e. $C \equiv 0$ ). The new problem is obtained by substituting the matrices $A, P, Q$ of the original problem with $\bar{A}, \bar{P}, \bar{Q}$ defined in (10), (13) and (14) and by substituting the infinite dimensional constraint (2) with

$$
D u \equiv 0 .
$$

Step 2. After the feed-back is applied the family of subspaces $H_{c}$ 's is now obtained by taking as parameter $c$ the time and by setting the control zero after $c$. By means of Assumption 2.2 we prove that $H_{c}$ satisfies the continuity properties, [20]. Moreover we prove that $J$ is elliptic if and only if the strengthened LegendreClebsh condition holds.

St ep 3. The zeros of $V$ are proved to correspond to non-trivial minima of $J$ restricted to $H_{c}$. The corresponding minimizers can be characterized by the solutions of the Jacobi system and by the transversality conditions induced by (11) and (12). These boundary conditions include an extra term which does not appear when the right end-point is fixed. The Jacobi system can be obtained by using, for example, Theorem 2.1 and the Legendre-Clebsch condition (Assumption 2.3) which allows us to express the control in terms of the trajectory and of the adjoint covector. The points in which $V$ changes sign are characterized by a result in [9] 
where it is stated that if the index changes at $c$, then there is an extremal in $H_{c}$ which is not extremal after $c$.

4. Final comments. In order to determine the accessory minimization problem, we reduce the optimal control problem to a minimization problem in a Banach space and then we find the extremals of the corresponding $C^{2}$ map which are characterized by abstract theorems on second derivatives. To follow this approach we need some regularity results on the flow of the control system and appropriate estimates on the remainder term. This approach allows us to obtain in an explicit form the multiplier relative to the infinite dimensional constraint. Moreover we can better understand the role of controllability and normality. In fact, most of the previous works assume controllability, while in order to obtain meaningful results normality (i.e. $\lambda_{0} \neq 0$ ) is sufficient. Normality is implied by controllability but it is not equivalent to it. In any case, it is interesting to underline that in the abnormal case we obtain information on the constraints (see Remark 2.1).

We need a surjectivity assumption on the state-control constraint (Assumption 2.2). If Assumption 2.2 is not satisfied, from the proof of Lemma 3.1 in [19] it follows that the codimension of the closure of the range of $\tilde{\chi}^{\prime}$ is infinite so that by using the Hahn-Banach Theorem we can prove that the space of multipliers is infinite dimensional and they belong to the dual of $L^{\infty}$.

The described results are a partial answer to the problems one can study in this subject. Further arguments of investigations are, for example, whether or not the same results hold true for $p$-WLM if we assume that all the data are polynomial in $u$ of degree $p$. A more interesting and difficult question concerns sufficient conditions for SLM. For this aim a generalization of the concept of field of extremals to this setting is needed.

Here we obtain that the infinite dimensional multiplier $\Lambda$ belongs to $L^{\infty}$. It would be interesting to prove that a Maximum Principle holds for the same multiplier. In [12] a Maximum Principle is derived but such a regularity of the multiplier is obtained by assuming a continuous $t$-dependence of the data.

The description of the properties of coerciveness and nonnegativity of the constrained quadratic form by means of (semi) conjugate points is complete. Let us remark that for the study of the signature of the quadratic form we do not need any kind of controllability with respect to the boundary conditions on the end-points, differently from all the other works on the subject. In fact, due to the linearity of the control system and of the constraints, the problem can be always considered normal.

\section{References}

[1] A. A. Agrachev, Quadratic mappings in geometric control theory, Itogi Nauki, Problemy Geometrii, 20 (1988), 111-205 (in Russian); English transl. in: J. Soviet Math. 51 (1990). 
[2] A. A. Agrachev and R. Gamkrelidze, Symplectic methods for optimization and control, in: B. Jakubczyk and W. Respondek (eds.), Geometry of Feedback and Optimal Control, Pure and Appl. Math., Marcel Dekker, New York, 1995.

[3] P. Bernhard, La théorie de la seconde variation et le problème linéaire quadratique, in: J. P. Aubin, A. Bensoussan and I. Ekeland (eds.), Advances in Hamiltonian Systems, Birkhäuser, Boston, 1983, 109-142.

[4] A. Dmitruk, The Euler-Jacobi equation in variational calculus, Mat. Zametki 20 (1976), 847-858 (in Russian); English transl.: Math. Notes 20 (1976), 1032-1038.

[5] A. L. Dontchev, W. W. Hager, A. B. Poore and B. Yang, Optimal stability and convergence in nonlinear control, preprint, 1992.

[6] W. H. Fleming and R. Rishel, Optimal Deterministic and Stochastic Control, Springer, Berlin, 1975.

[7] E. G. Gilbert and D. S. Bernstein, Second order necessary conditions in optimal control: accessory-problem results without normality conditions, J. Optim. Theory Appl. 41 (1983), 75-106.

[8] K. A. Grasse, Controllability and accessibility in nonlinear control systems, $\mathrm{PhD}$ thesis, University of Illinois at Urbana-Champaign, 1979.

[9] M. R. Hestenes, Applications of the theory of quadratic forms in Hilbert space to calculus of variations, Pacific J. Math. 1 (1951), 525-581.

[10] -, Calculus of Variations and Optimal Control Theory, Wiley, New York, 1966.

[11] E. Levitin, A. Milyutin and N. Osmolovskǐ̌, Conditions of high order for a local minimum in problems with constraints, Uspekhi Mat. Nauk, 33 (1978), 85-148 (in Russian); English transl.: Russian Math. Surveys 33 (1978), 97-168.

[12] K. Makowski and L. Neustadt, Optimal control problems with mixed control-phase variable equality and inequality constraints, SIAM J. Control 12 (1974), 184-228.

[13] H. Maurer, First and second order sufficient optimality conditions in mathematical programming and optimal control, Math. Programming Stud. 14 (1981), 163-177.

[14] J. Mawhin and M. Willem, Critical Point Theory and Hamiltonian Systems, Springer, New York, 1989.

[15] E. Mikami, Focal points in a control problem, Pacific J. Math. 35 (1970), 473-485.

[16] M. Morse, The Calculus of Variations in the Large, Amer. Math. Soc. Colloq. Publ. 18, New York, 1934.

[17] N. Osmolovskiı̌, Second order conditions for a weak local minimum in an optimal control problem (necessity, sufficiency), Dokl. Akad. Nauk SSSR 225 (1975) (in Russian); English transl.: Soviet Math. Dokl. 16 (1975), 1480-1483.

[18] G. Stefani and P. Zezza, A new type of sufficient optimality conditions for a nonlinear constrained optimal control problem, in: M. Fliess (ed.), Proceedings of the Nonlinear Control System Design Symposium, Bordeaux, 1992, 713-719.

[19] - - - Optimal control problems with mixed state-control constraints: necessary conditions, J. Math. Syst. Est. Control 2 (1992), 155-189.

[20] - - - The Jacobi condition for LQ-control problems with constraints, in: J. W. Nieuwenhuis, C. Praagman and H. L. Trentelman (eds.), Proceedings of the Second European Control Conference, 1993, 1003-1007.

[21] - - - Optimality conditions for a constrained optimal control problem, preprint, University of Florence, DiMaDEFAS, 1993

[22] - - - Regular constrained LQ-control problems, preprint \#12-94, University of Florence, DiMaDEFAS.

[23] J. Warga, Second order necessary conditions in optimization, SIAM J. Control Optim. 22 (1984), 524-528.

[24] V. Zeidan, Sufficiency conditions for variational problems with variable endpoints: coupled points, Appl. Math. Optim. 27 (1993), 191-209. 
[25] V. Zeidan and P. Zezza, Necessary conditions for optimal control problems: conjugate points, SIAM J. Control Optim. 26 (1988), 592-608.

[26] - - - Coupled points in optimal control, IEEE Trans. Automat. Control 36 (1991), 1276-1281.

[27] P. Zezza, The Jacobi condition for elliptic forms in Hilbert spaces, J. Optim. Theory Appl. 76 (1993), 357-380. 